\title{
Secondary transmissions during the outbreak of Shiga toxin- producing Escherichia coli O104 in Hesse, Germany, 2011
}

\author{
A M Hauri (anja.hauri@hlpug.hessen.de) ${ }^{1}$, U Götsch ${ }^{2}$, I Strotmann ${ }^{3}$, J Krahn ${ }^{4}$, G Bettge-Weller ${ }^{1}$, H J Westbrock ${ }^{1}, 0$ Bellinger ${ }^{2}$, \\ H Uphoff ${ }^{1}$ \\ 1. Hesse State Health Office, Dillenburg, Germany \\ 2. Public Health Authority, Frankfurt, Germany \\ 3. Public Health Authority, Kassel, Germany \\ 4. Public Health Authority, Darmstadt-Dieburg, Germany
}

Hauri AM, Götsch U, Strotmann I, Krahn J, Bettge-Weller G, Westbrock HJ, Bellinger O, Uphoff H. Secondary transmissions during the outbreak of Shiga toxin-producing Escherichia coli 0104 in Hesse, Germany, 2011.

Euro Surveill. 2011;16(31):pii=19937. Available online: http://www.eurosurveillance.org/ViewArticle.aspx?Articleld=19937

Article published on 4 August 2011

During the recent outbreak of Shiga toxin-producing Escherichia coli (STEC) 0104: 44 in Germany most cases notified in the State of Hesse (6 million inhabitants) were linked to satellite clusters or had travelled to the outbreak area in northern Germany. Intensified surveillance was introduced to rapidly identify cases not linked to known clusters or cases and thus to obtain timely information on possible further contaminated vehicles distributed in Hesse, as well to describe the risk of secondary transmission among known cases. As of 2 August 2011*, 56 cases of haemolytic uraemic syndrome (HUS) including two fatal cases, and 124 cases of STEC gastroenteritis meeting the national case definitions have been reported in Hesse. Among the 55 HUS and 81 STEC gastroenteritis cases that met the outbreak case definition, one HUS case and eight STEC gastroenteritis cases may have acquired their infection through secondary transmission. They include six possible transmissions within the family, two possible nosocomial and one possible laboratory transmission. Our results do not suggest an increased transmissibility of the outbreak strain compared to what is already known about $E$. coli 0157 and other STEC serotypes.

\section{Introduction}

On 19 May 2011, the public health authority of Frankfurt, Hesse, and the Robert Koch Institute (RKI), Germany's national public health authority, were informed about clusters of cases of haemolytic-uraemic syndrome (HUS) in Frankfurt and Hamburg [1,2]. These were the first notified cases of an outbreak of Shiga toxinproducing Escherichia coli (STEC) serotype 0104: $\mathrm{H}_{4}$. Between 1 May and 20 July 2011, 727 HUS cases and 3,039 STEC cases with diarrhoea have been reported in Germany [3]. Epidemiological evidence suggested that STEC-contaminated sprouts were the vehicle of infection. Trace back studies carried out by the German Enterohaemorrhagic E. coli Task Force and authorities of Lower Saxony identified one sprout-producing farm in Lower Saxony (Establishment A) as being the most likely source of sprouts contaminated with STEC 0104 . In a second step, forward tracing established that all 41 case clusters identified at that time in Germany were linked to consumption of sprouts originating from Establishment A [4].

The outbreak strain has been microbiologically characterised in detail [5]: All outbreak strains investigated belonged to serotype $0_{104}: \mathrm{H}_{4}$ and contained the $s x_{2}$ gene, encoding Shiga toxin 2 (Stx2). The eae gene, encoding adhesion intimin, and ast $A$, encoding enteroaggregative $E$. coli Shiga toxin 1 (Stx1) were not present. All isolates displayed an extended-spectrum beta-lactamase (ESBL) phenotype.

The highest HUS incidences were reported from the northern German states of Hamburg, SchleswigHolstein, Bremen, Mecklenburg-Vorpommern and Lower Saxony, the so-called northern German outbreak area. Aside from satellite clusters in Hesse and eastern North Rhine-Westphalia, most of the HUS cases from other states could be linked to travel-related exposure in the outbreak area [1].

Identified clusters in Hesse included patrons of cafeterias run by Company $A$ and guests of two private parties. Sprouts served in the cafeterias and sprouts used in a salad brought by one of the guests from northern Germany to Party A were traced back to Establishment A. Foods supplied to Party B were prepared by a caterer who is likely to have acquired the infection through person-to-person transmission.

The first possible secondary cases were reported in Hesse at the end of May, and local public health authorities were requested on 1 June 2011 to systematically collect and report information on possible secondary cases to the Hessian state health office. Starting on 7 June, testing for the outbreak strain of stool samples from patients notified with STEC infection and from symptomatic household members of outbreak cases 
was offered free of charge at the state health office and performed according to RKI recommendations [6]. The aim was to rapidly identify cases not linked to known clusters and thus obtain timely information on possible further contaminated vehicles distributed in Hesse, as well as to describe the risk of secondary transmission among known cases.

Here we present data on reported cases of STEC gastroenteritis and HUS in Hesse with symptom onset since 1 May 2011. We provide additional information on possible secondary outbreak cases and on cases without an epidemiological link to identified clusters or known outbreak cases.

\section{Methods}

Hesse is one of the 16 German states, with a population of 6.0 million, subdivided into 26 counties.

We extracted from the Hessian database for notifiable diseases all cases of STEC gastroenteritis and HUS meeting the national case definitions with disease onset on or after 1 May 2011. Data were extracted as of 2 August 2011 and further updates are to be expected. Disease onset was defined as the onset of diarrhoea, regardless of whether the HUS developed at a later date. We summarised data available at local public health authorities on exposures of cases, including possible epidemiological links to known cases or clusters, and on laboratory reports. For (possible) outbreak cases without epidemiological link (as defined below) we contacted the primary diagnosing laboratory or the national reference or consulting laboratories to obtain additional information on diagnostic tests done and their results.

Data analysis was done with STATA (StataCorp LP, United States, version 11.2). For statistical comparisons the Mann-Whitney $\mathrm{U}$ test was used for age distribution and the Pearson chi-square test for proportions.

\section{STEC gastroenteritis and HUS case definitions} of the German surveillance system

According to the German Protection against Infection Act of 2001, the detection of a Shiga toxin (Stx) in E. coli

\section{FIGURE 1}

STEC gastroenteritis and HUS cases and criteria for sporadic cases and outbreak cases with or without epidemiological link, Hesse, Germany, 1 May-2 August* $2011(n=180)$

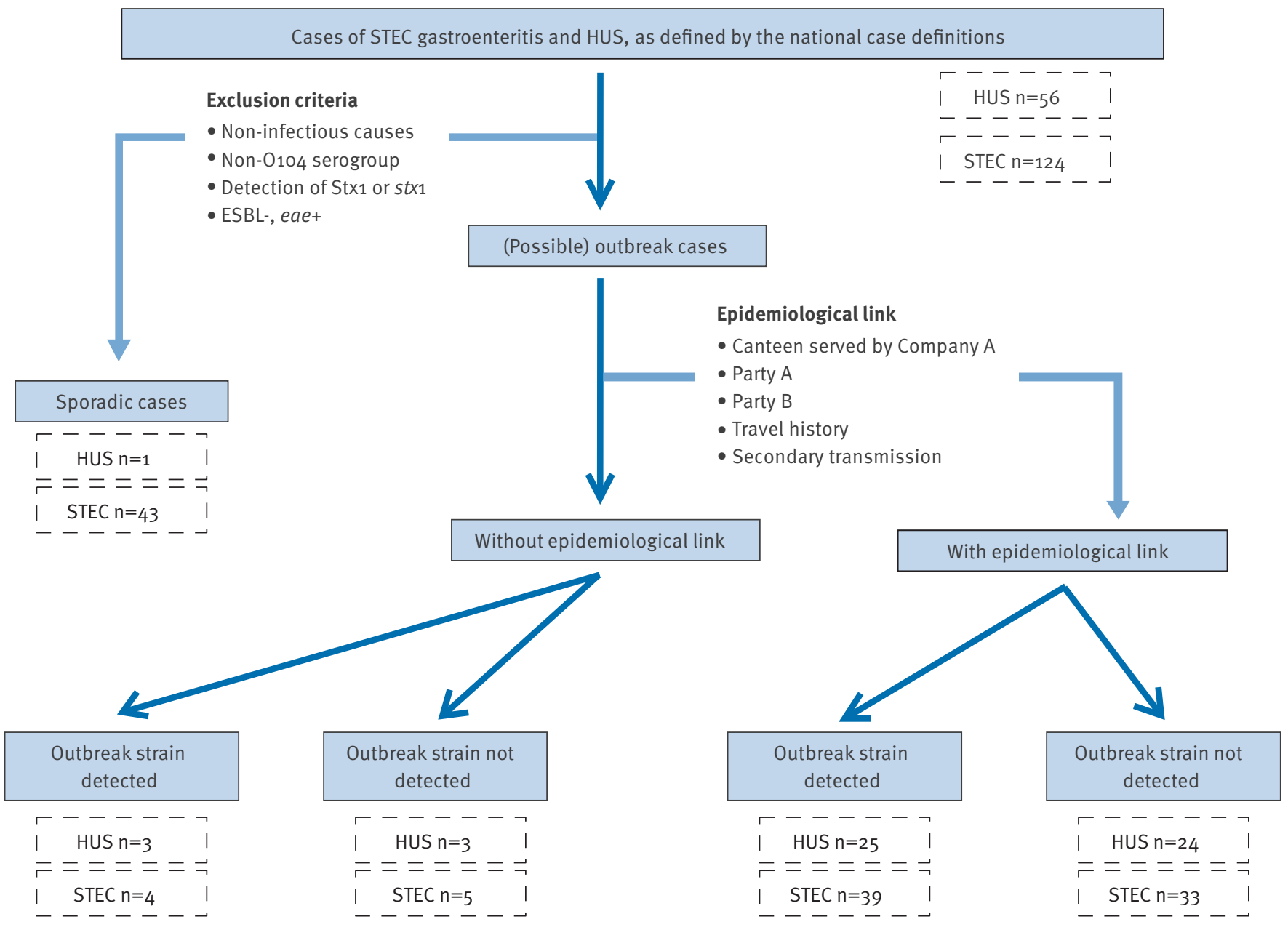

Eae: adhesion intimin; ESBL: extended-spectrum beta-lactamase; HUS: haemolytic uraemic syndrome; STEC: Shiga toxin-producing Escherichia coli; Stx: Shiga toxin. 
isolates or of a Shiga toxin gene (stx) in stool enrichment culture or isolates must, by law, be reported by diagnosing laboratories to local health departments [2]. The German case definition of STEC gastroenteritis (without HUS) requires the presence of at least one of the following symptoms: diarrhoea (three or more loose stools in a 24-hour period), abdominal cramps, or vomiting in addition to a laboratory confirmation (as defined above) or an epidemiological link to laboratory-confirmed case. Physicians are required to report clinical symptoms compatible with diarrhoea-associated HUS in a patient. The German case definition of HUS comprises thrombocytopenia (platelet count of $\left\langle 150,000\right.$ per $\mathrm{mm}^{3}$ ), haemolytic anaemia, and acute renal dysfunction [7]. Reported cases of HUS or STEC infection are investigated and recorded by the local health department and, if case definitions are met, the reports are forwarded electronically, without identifying information, through the state to the federal level.

\section{Outbreak case definitions}

For cases fulfilling the case definition for STEC gastroenteritis or HUS we further distinguished between sporadic cases and outbreak cases. To define sporadic cases we used a set of exclusion criteria based on laboratory results $[8,9]$ : detection of a non-0104 serogroup, of Stx1 or its encoding gene $s t x_{1}$, detection of eae, or of an E. coli strain not displaying an ESBL phenotype. Therefore, by definition, outbreak cases included possible outbreak cases, i.e. cases without any epidemiological link to known cases and for which the outbreak strain could not be detected in a stool sample (Figure 1).
Outbreak cases were considered epidemiologically linked if they were patrons of a canteen served by Company A, guests of Party A or B, if they had travelled to the northern German outbreak area during their incubation period or were linked to an STEC 0104:H4-cluster outside Hesse, or if they were thought to have acquired their infection through secondary transmission. Secondary transmissions included contacts of epidemiologically linked persons as defined above and possible nosocomial and laboratory transmission.

For surveillance purposes, the RKI defined combinations of at least two laboratory results to be sufficiently specific for the outbreak strain [9]. For example, in case of detection of the $s x_{2}$ gene in an ESBL-positive isolate or detection of $s t x_{2}$ gene and serotype $0_{104}$, detection of the outbreak strain was assumed. The RKI requested all local public health authorities to interpret laboratory results and to forward reports accordingly.

\section{Results}

As of 2 August $2011^{\star}, 56$ HUS cases, including two fatal cases, and 124 STEC gastroenteritis cases meeting the national case definitions were reported in Hesse, with onset dates of 1 May or later (Figures 2 and 3). Of these, 55 HUS cases and 81 STEC gastroenteritis cases met the outbreak case definitions (Figure 1).

Among the 55 HUS outbreak cases, 49 were epidemiologically linked: 27 cases linked to Company A, two cases to Party A, two cases to Party B, 17 cases with travel history and one case of secondary transmission.

\section{FIGURE 2}

Epidemic curve of HUS cases meeting the case definition for (possible) outbreak cases, Hesse, Germany, 1 May-21 July 2011 $(\mathrm{n}=55)$

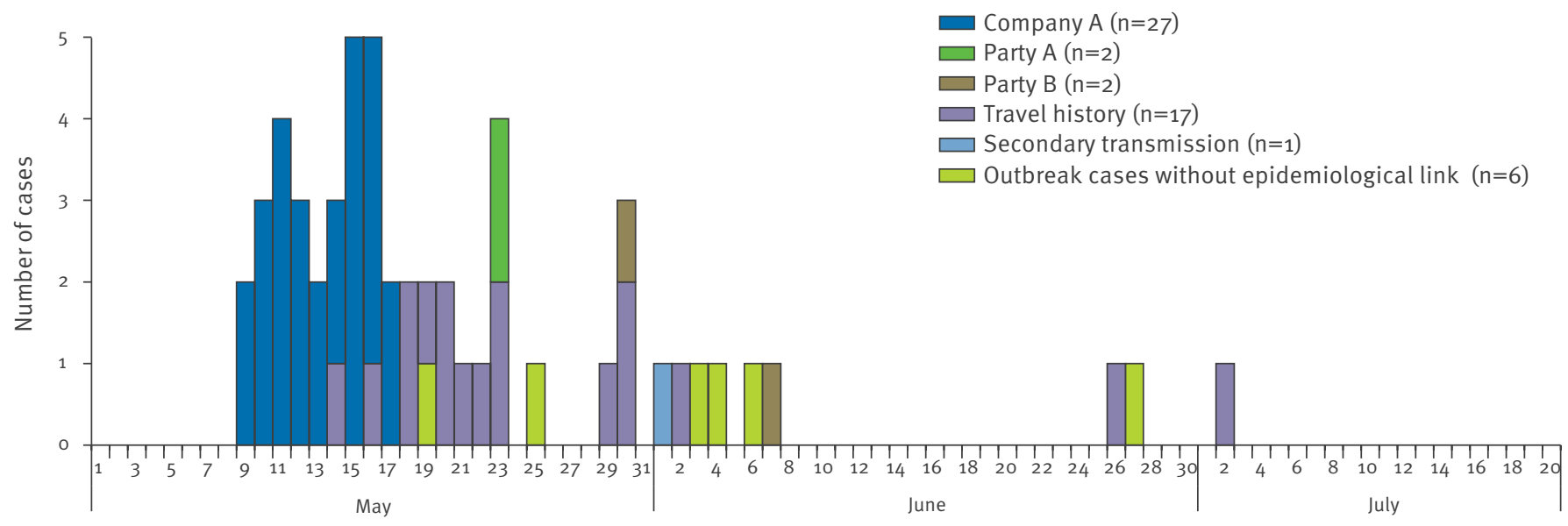

Date of symptom onset 2011

HUS: haemolytic uraemic syndrome.

(Possible) outbreak cases include epidemiologically linked cases (canteen served by Company A, Party A and B, exposure history to the northern German outbreak area, secondary transmission) and epidemiologically unlinked cases. Date of hospitalisation was used when date of onset of diarrhoea was not available. 
The age of epidemiologically linked HUS cases ranged from 21 to 75 years (median: 39 years). The six epidemiologically unlinked HUS cases were 5, 7, 41, 42, 64 and 73 years-old. Thirty-four of the 55 cases were female. The sporadic case was a four year-old girl with STEC O157 infection.

Among 81 STEC gastroenteritis outbreak cases, 72 were epidemiologically linked to the outbreak: 27 cases linked to Company A, no case at Party A, eight cases at Party B, 29 cases with travel history and eight cases with possible secondary transmissions. For 43 of the 81 cases the outbreak strain could be detected in a stool sample (Figure 1). Sporadic cases had symptom onsets from 3 May until 8 July 2011. For 15 of the 43 sporadic cases information on the identified serotype was available: five were serotype 0157 , three were serotype 091, and two were serotype 103. The median age of all patients reported to have STEC gastroenteritis was 44 years and did not differ significantly between outbreak and sporadic cases ( 44 and 42 years, respectively). Among the STEC gastroenteritis cases, 44 of 77 outbreak cases and 26 of the 43 sporadic cases were female. Information on sex was missing for four outbreak cases.

The outbreak strain was detected in stool samples of four of the nine epidemiologically unlinked STEC gastroenteritis cases. They are described below together with the unlinked HUS cases.
Cases with epidemiological link: possible secondary transmissions

Among outbreak cases, eight of the 81 STEC gastroenteritis cases and one of the 55 HUS case were possible secondary cases. They included six transmissions within the family, two nosocomial and one laboratory transmission. The strength of the epidemiological and laboratory evidence linking these cases to their respective index cases or the known clusters differs. Therefore, these possible secondary transmissions are described in detail.

\section{Family 1}

On 24 May 2011, a woman in her 40s, whose husband had eaten at a cafeteria served by Company A, fell sick with bloody diarrhoea and stomach cramps. She was hospitalised on 26 May 2011 and subsequently the outbreak strain was isolated from a stool sample. On 27 May 2011, the local public health authority took stool samples from the husband, and the one and eight yearold children. Stool samples were tested in a private microbiology laboratory using broth enrichment culture for STEC and an ELISA test for Stx1/2. They were repeatedly negative for the husband and the eight yearold child. Stool samples of the one year-old child had a positive ELISA Stx1/2 result in all three samples. No further laboratory tests were done. The father reported light stomach pain but no diarrhoea on 18 May 2011 and for the one year-old child light non-bloody diarrhoea some time before symptom onset of the mother. No foods sold at the Frankfurt canteen were eaten by

\section{FIGURE 3}

Epidemic curve of STEC gastroenteritis cases meeting the case definition for (possible) outbreak cases, Hesse, Germany, 1 May-21 July $2011(\mathrm{n}=81)$

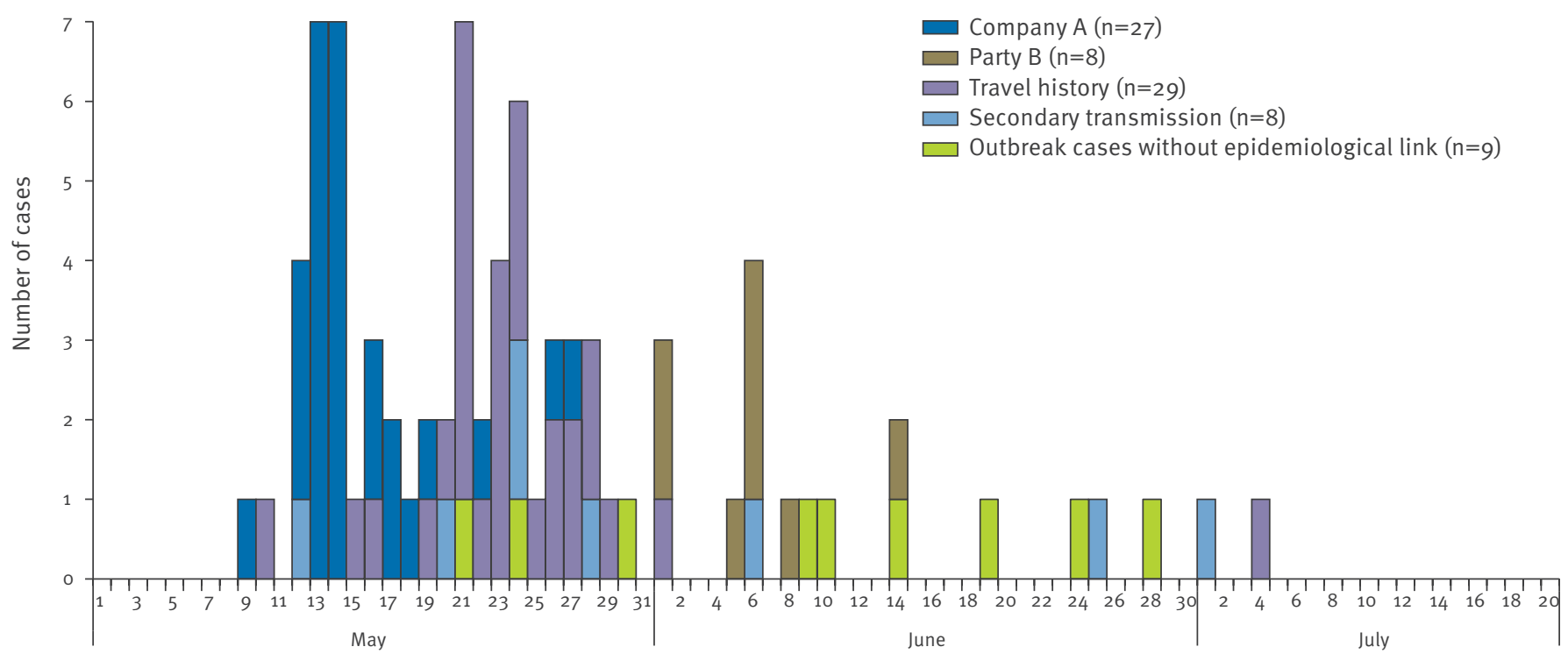

Date of symptom onset 2011

STEC: Shiga toxin-producing Escherichia coli.

(Possible) outbreak cases include epidemiologically linked cases (canteen served by Company A, Party B, exposure history to the northern German outbreak area, secondary transmission) and epidemiologically unlinked cases. Date of hospitalisation was used when date of onset of diarrhoea was not available. 
the mother and the two children and no travel to other outbreak areas was reported.

Family 2

A woman in her 30 s fell ill on 12 May 2011 with bloody diarrhoea and was hospitalised on the same day. During her hospital stay she had a colonoscopy, which included clearing of the colon of solid matter. She was discharged on 14 May and subsequently readmitted on 23 May 2011. Starting on 25 May 2011, three stool samples were taken, but all tested negative for STEC. She and her husband had eaten meals at a canteen served by Company A during the two weeks before symptom onset. The husband and a two year-old child did not report any gastrointestinal symptoms. After being discharged from the hospital the family left for vacation and no further stool samples could be taken before their departure.

The woman's mother was hospitalised on 1 June 2011 for HUS. The outbreak strain was isolated from a stool sample. She had visited her daughter during her first hospital stay and taken case of her two year-old grandchild on five days from 16 to 25 May 2011. The grandmother attended the child in the household of her daughter, who reported having used a separate toilet.

The grandmother's husband fell ill with diarrhoea on 6 June 2011 and was hospitalised the following day. The outbreak strain was isolated from a stool sample. He had not visited his daughter or grandchild. He and his wife had not travelled to northern Germany.

\section{Family 3}

On 14 May 2011, a woman in her 40 s living in an On 14 May 2011, a woman in her 40 s living in an assisted accommodation became ill with bloody diarrhoea and was hospitalised for HUS on 17 May 2011. She had eaten meals prepared in a cafeteria served by Company $A$. Her mother, a woman in her 70s, assisted in caring for her in the first days after symptom onset. The mother fell sick with bloody diarrhoea on 28 May 2011 and was hospitalised on the same day. The outbreak strain was isolated from stool samples from both patients.

\section{Family 4}

A woman in her 20 s became ill with bloody diarrhoea on 10 May and subsequently developed HUS. She had eaten meals from Company A during the two weeks before symptom onset. On 13 May 2011 she moved to the house of her mother, who took care of her until the daughter's hospitalisation on 15 May. The mother, in her 50s, developed bloody diarrhoea on 24 May 2011 and was hospitalised on 26 May 2011 for STEC gastroenteritis. The outbreak strain was isolated from stool samples from both patients.

Nosocomial transmission 1

A man in his 70 secame ill with bloody diarrhoea on 28 May 2011. E. coli was isolated from a stool sample and confirmed as the outbreak strain at the national reference centre. The patient had been hospitalised from 12 to 16 May 2011 with a diagnosis of diverticulitis. He reported generally eating only at home. During the incubation period he had not eaten sprouts and not travelled to northern Germany. He did not know of any diarrhoeal illness among his family members or acquaintances or any link to known clusters or the outbreak-associated cafeterias. No further outbreak cases are known to have been hospitalised on the same ward or among the staff. However, given the long incubation period of the outbreak strain, nosocomial transmission cannot be excluded with certainty.

Nosocomial transmission 2

A woman in her 30 s was hospitalised until 10 June 2011 for a neurological diagnosis unrelated to the STEC outbreak. She had had meals in a canteen served by Company A and became ill on 17 May 2011 with STEC gastroenteritis. Isolation precautions were followed in the hospital, given that at the time of hospitalisation she was a known asymptomatic carrier of the outbreak strain. Nevertheless, the patient once spread faeces on the ward during a delirious episode. A man in his 205 was an inpatient of the same ward on 9 and 10 June 2011. He continued to be hospitalised until 25 June 2011 when he developed bloody diarrhoea. An $s t x_{2}+$, st $x_{1}-E$. coli of an ESBL phenotype was isolated from a stool sample and confirmed as the outbreak strain at the national reference centre.

\section{Laboratory infection}

A woman in her 20 s fell ill with bloody diarrhoea on 1 July 2011. She had been in contact with the outbreak strain during the incubation period while working in a microbiology laboratory. The outbreak strain was isolated from her stool sample. She had not travelled to northern Germany and not eaten sprouts during the incubation period. She lives in an area without known outbreak clusters and had no known link to Company $A$ or the two private parties.

\section{Cases without epidemiological link}

Among outbreak cases, nine of the 81 STEC gastroenteritis cases and six of the 55 HUS cases had no epidemiological link to known clusters or possible secondary cases (Figures 1-3). Among epidemiologically unlinked outbreak cases, the outbreak strain was detected in stool samples of three HUS and four STEC gastroenteritis cases. The three HUS cases fell ill on 19 and 25 May and 6 June 2011. They were 7, 42 and 73 yearsold. None of them had recently travelled to northern Germany or had any known contact to outbreak cases or known clusters. Only one of them reported having eaten sprouts once during the incubation period. The four STEC gastroenteritis cases with the outbreak strain detected in a stool sample fell ill on 21 May, and on 9, 24 and 28 June 2011. They were 10, 24, 32 and 55 years-old. None of them reported having eaten sprouts, any recent travel to northern Germany or known contact to outbreak cases or known clusters. Of the seven epidemiologically unlinked cases with the outbreak 
strain detected in a stool sample, five live (four cases) or work (one case) in the two cities in Hesse with the highest incidences of outbreak cases.

\section{Discussion}

As of 2 August 2011, a total of 55 HUS and 124 STEC gastroenteritis outbreak cases have been reported in Hesse. Among these cases, at least nine cases may have acquired their disease through transmissions within the family, nosocomial or laboratory transmission. These nine cases are a minimum estimate of possible secondary transmissions. Given the long incubation period of this pathogen (median eight days) [2], distinction between co-primary cases and secondary transmission is difficult for family members with a common exposure history. Whenever we were unable to distinguish between co-primary and secondary person-to-person transmission, cases were categorised as co-primary, i.e. epidemiologically linked to the northern German outbreak area, cafeterias served by Company A, or to Party A or B. Therefore, while misclassification of secondary cases as co-primary cases is possible, we know of no cases that occurred more than 10 days apart among family members linked to the northern German outbreak area or the two private parties. In addition, risk of secondary transmissions within Hesse may have been reduced if travel-associated cases became sick and were hospitalised while still on travel outside Hesse. It has previously been shown that hospitalisation of STEC cases reduces the risk of household transmissions $[10,11]$.

All six transmissions within the family described here were linked to Company A. Three of these six transmissions occurred among non-regular household members, i.e. family members who had moved in temporarily to provide or receive assistance during sickness. Many cases linked to cafeterias served by Company $A$ live in small households of one or two persons and without children. This may have contributed to limiting the number of secondary cases, especially among children. The presence of siblings and the young age of the index cases has been associated with increased transmission risk [10-12], and transmissions between families have been described previously in outbreak settings [13].

While the outbreak strain was present in stool samples of five secondary cases within families, it could only be detected in stool samples of three of the four respective index cases. In Family 1, the index case - who had eaten at a cafeteria served by Company $A$ - reported only light stomach pain for one day during the two weeks before symptom onset of his wife. It remains unclear if the stomach pain was related to an STEC infection, if he had an asymptomatic STEC infection, or if - in our view less likely - he was not infected. The first cases linked to Company A fell ill on 9 May 2011 and the index case's stool samples may have become negative by the time they were first tested (on 27 May). For non-outbreak STEC infections, identification in patient's faeces late in the illness has been shown to be difficult [14].

Secondary transmissions frequently occur in outbreaks caused by $E$. coli 0157 [15] and have been described to occur in $4-15 \%$ of households following sporadic infection [11]. In a population-based study in Scotland, $11 \%$ of 0157 cases were identified as secondary [12]. In addition, nosocomial and laboratory-acquired infections with $E$. coli 0157 have been reported $[16,17]$. They underline the need for strict adherence to standard infection control precautions [18].

Several episodes of secondary transmissions and asymptomatic carriage have already been described for the recent 0104 outbreak in Germany [19-21]. In the three instances where we could calculate a serial interval, the time span between symptom onset of primary and secondary cases was 14,14 and 20 days, confirming previous reports for the outbreak strain [21] and for E. coli $\mathrm{O}_{157}$ [10] that, considering the relatively long incubation time for the outbreak strain, household transmission occurs early during disease.

We have here described seven epidemiologically unlinked cases for whom the outbreak strain could be detected in a stool sample. Several possibilities may explain these seven cases: (i) our definition of outbreak strain may have been too generic, i.e. the $E$. coli strains identified may not have been outbreak strains, (ii) these infections may have been acquired from foodborne transmission, and (iii) secondary transmission. Several of the laboratory results for the seven epidemiologically unlinked cases have been confirmed by the national reference and consulting laboratories (while others are pending) and we have no further evidence suggesting that contaminated foods were circulating in Hesse outside the identified clusters. Direct or indirect secondary transmission among non-close contacts may therefore be the most likely explanation for most of these seven cases.

We extracted from the Hessian database for notifiable diseases only data on cases meeting the national case definitions for STEC gastroenteritis and HUS. Data cleaning and analysis on asymptomatic cases and on cases with symptoms not meeting the national case definition (e.g. only one episode of loose stool) from the restaurant outbreaks is still ongoing.

From 3 May to 8 July 2011, 43 non-outbreak cases of STEC gastroenteritis were reported in Hesse. In comparison, during the five-year period from 2006 to 2010 , only 76 cases of STEC gastroenteritis were reported. Serogroup 0157 was the most commonly detected serogroup in the European Union in 2008 and 2009, representing about $52 \%$ of STEC cases with known serotypes [22]. Reported cases represent a subset of infections in the community [23] and testing for STEC infection increased considerably during the outbreak. Therefore, in outbreak settings, the timely distinction 
between sporadic and outbreak cases is important in orienting further investigations and control measures of public health and veterinary authorities.

The current outbreak strain is a very rare serogroup in humans in Europe and worldwide [22]. This and its other unique characteristics may be part of the reason why the possible nosocomial and laboratory infections were identified and why we considered the unlinked cases as probable secondary transmissions. Adult age of index patients and transmission among non-regular household members are particular characteristics of the described secondary transmissions. They should not be interpreted as indicative of a particular high transmissibility of the outbreak strain. The majority of the transmissions involve patients residing in different counties. We believe that the particular characteristics of the outbreak strain together with the structure of the German surveillance system (including local and state levels and a national level) facilitated the identification and description of possible secondary transmissions.

In conclusion, the outbreak strain can be easily transmitted but our preliminary results do not suggest an increased transmissibility of the outbreak strain compared to what is already known about E. coli 0157 and other STEC serotypes.

\section{*Authors' correction:}

On request of the authors the phrase "As of 21 July" was changed to "As of 2 August" in the abstract and the first sentence of the results. This date was also corrected in the title of Figure 1. These changes were made on 1 Sept 2011.

\section{Acknowledgements}

We are especially grateful to our colleagues at the Hessian public health and veterinary authorities for their contributions. We would also like to thank all laboratories who provided information on procedures followed and their results.

\section{References}

1. Frank C, Faber MS, Askar M, Bernard H, Fruth A, Gilsdorf $A$, et al. Large and ongoing outbreak of haemolytic uraemic syndrome, Germany, May 2011. Euro Surveill. 2011;16(21):pii=19878. Available from: http://www. eurosurveillance.org/ViewArticle.aspx?Articleld $=19878$

2. Frank C, Werber D, Cramer JP, Askar M, Faber M, an der Heiden $M$, et al. Epidemic Profile of Shiga-Toxin-Producing Escherichia coli 0104:H4 Outbreak in Germany - Preliminary Report. N Engl J Med. 2011 Jun 22. [Epub ahead of print].

3. Robert Koch-Institute (RKI). EHEC/HUS 0104:H4 - The outbreak is considered to be over. 29 Jul 2011. Berlin: RKI; 29 Jul 2011. Available from: http://www.rki.de/cln_117/nn_205760/EN/ Home/homepage__node.html?_nnn=true

4. European Food Safety Authority (EFSA): Tracing seeds, in particular fenugreek (Trigonella foenum-graecum) seeds, in relation to the Shiga toxin-producing E. coli (STEC) $0_{104}: \mathrm{H}_{4}$ 2011 Outbreaks in Germany and France. Parma: EFSA; 2011. Available from: http://www.efsa.europa.eu/en/supporting/ doc/176e.pdf

5. Bielaszewska M, Mellmann A, Zhang W, Köck R, Fruth A, Bauwens A, et al. Characterisation of the Escherichia coli strain associated with an outbreak of haemolytic uraemic syndrome in Germany, 2011: a microbiological study. Lancet Infect Dis. 22 Jun 2011. [Epub ahead of print]
6. Robert Koch Institut (RKI). EHEC 0104: $\mathrm{H}_{4}$ - Eigenschaften des Erregers sowie Hinweise und Hilfestellungen des RKI zur Diagnostik des gegenwärtig zirkulierenden Ausbruchstammes. [EHEC 0104: $\mathrm{H}_{4}$ - characteristics of the pathogen and information and assistance of the RKI in the diagnosis of the currently circulating outbreak strain]. Berlin: RKI; 24 Jun 2011. German. Available from: http://www.rki.de/cln_151/ nn_467482/DE/Content/InfAZ/E/EHEC/EHEC_Diagnostik,temp lateld=raw, property=publicationFile.pdf/EHEC_Diagnostik.pdf

7. Robert Koch Institute (RKI). Falldefinitionen des Robert KochInstituts zur Übermittlung von Erkrankungs- oder Todesfällen und Nachweisen von Krankheitserregern. [Case definitions for the surveillance of notifiable infectious diseases in Germany ]. Berlin: RKI; 2007. [Accessed 15 June 2011]. German. Available from: http://www.rki.de/cln_178/nn_200710/DE/Content/ Infekt/IfSG/Falldefinition/Falldefinition,templateld=raw, proper ty=publicationFile.pdf/Falldefinition.pdf

8. Robert Koch Institute (RKI). [Case definition for HUS-cases associated with the outbreak in the spring 2011 in Germany 2011]. RKI. 1 Jul 2011. German. Available from: http://www. rki.de/cln 178/nn 467482/DE/Content/InfAZ/E/EHEC/ Falldefinition.htm

9. Robert Koch Institut (RKI). Themen zum Meldewesen. [Case reporting]. Infobrief 35. Berlin: RKI; 4 Jul 2011. German.

10. Werber D, Mason BW, Evans MR, Salmon RL. Preventing household transmission of Shiga toxin-producing Escherichia coli 0157 infection: promptly separating siblings might be the key. Clin Infect Dis. 2008;46(8):1189-96.

11. Parry SM, Salmon RL. Sporadic STEC 0157 infection: secondary household transmission in Wales. Emerg Infect Dis. 1998;4(4):657-61.

12. Locking ME, Pollock KG, Allison LJ, Rae L, Hanson MF, Cowden JM. Escherichia coli 0157 infection and secondary spread, Scotland, 1999-2008. Emerg Infect Dis. 2011;17(3):524-7.

13. Sayers G, McCarthy T, O’Connell M, O’Leary M, O’Brien D, Cafferkey $M$, et al. Haemolytic uraemic syndrome associated with interfamilial spread of E. coli $\mathrm{O}_{26} \mathrm{H}$ 11. Epidemiol Infect. 2006;134(4):724-8.

14. Mellmann A, Bielaszewska M, Zimmerhackl LB, Prager R, Harmsen D, Tschäpe H, et al. Enterohemorrhagic Escherichia coli in human infection: in vivo evolution of a bacterial pathogen. Clin Infect Dis. 2005;41(6):785-92.

15. Snedeker KG, Shaw DJ, Locking ME, Prescott RJ. Primary and secondary cases in Escherichia coli 0157 outbreaks: a statistical analysis. BMC Infect Dis. 2009;9:144.

16. Weightman NC, Kirby PJ. Nosocomial Escherichia coli 0157 infection. J Hosp Infect. 2000; 44(2):107-11.

17. Spina N, Zansky S, Dumas N, Kondracki S. Four laboratoryassociated cases of infection with Escherichia coli 0157: $\mathrm{H} 7$. J Clin Microbiol. 2005;43(6):2938-9.

18. Weber DJ, Rutala WA. The emerging nosocomial pathogens Cryptosporidium, Escherichia coli $\mathrm{O}_{157} \mathrm{H7}$, Helicobacter pylori, and hepatitis C: epidemiology, environmental survival, efficacy of disinfection, and control measures. Infect Control Hosp Epidemiol. 2001;22:306-15.

19. Kuijper EJ, Soonawala D, Vermont C, van Dissel JT. Household transmission of haemolytic uraemic syndrome associated with Escherichia coli 0104: $\mathrm{H}_{4}$ in the Netherlands, May 2011. Euro Surveill. 2011;16(25):pii=19897. Available from: http://www. eurosurveillance.org/ViewArticle.aspx?Articleld=19897

20. European Centre for Disease Prevention and Control (ECDC). Shiga toxin-producing E. coli (STEC): Update on outbreak in the EU, 8 July 2011. Epidemiological updates. Stockholm: ECDC. Available from: http://ecdc.europa.eu/en/activities/sciadvice/ Lists/ECDC \% 20Reviews/ECDC_DispForm.aspx?List $=512 \mathrm{ff} 74 \mathrm{f} \%$ 2D77d4\%2D4ad8\%2Db6d6\%2Dbfof 23083f30\&ID=1145\&RootF older $=\% 2$ Fen $\% 2$ Factivities $\% 2$ Fsciadvice $\% 2$ FLists $\% 2$ FECDC $\% 20$ Reviews

21. Institut de Veille Sanitaire (InVS). Bilan final sur les cas groupés d'infections à Escherichia coli entéro-hémorragique (EHEC) en Gironde, 26 juillet 2011. [Final balance of clusters of infection with enterohaemorrhagic Escherichia coli (EHEC) in Gironde, 26 July 2011]. Paris: InVS. French. Available from: http://www.invs.sante.fr/Dossiers-thematiques/Maladiesinfectieuses/Risques-infectieux-d-origine-alimentaire/ Syndrome-hemolytique-et-uremique/Actualites

22. European Centre for Disease Prevention and Control and European Food Safety Authority (ECDC/ EFSA) joint technical report: Shiga toxin/verotoxin-producing Escherichia coli in humans, food and animals in the EU/EEA, with special reference to the German outbreak strain STEC 0104 Stockholm: ECDC; 2011. Available from: http://www.ecdc. europa.eu/en/publications/Publications/1106_TER_EColi_ joint EFSA.pdf

23. Hauri AM, Uphoff H, Gawrich S. [The burden of acute gastrointestinal illness in Hesse--a telephone survey]. Gesundheitswesen. 2011;73(2):78-84. German.. 